\title{
Asynchronous $H_{\infty}$ Fuzzy Control for A Class of Switched Nonlinear Systems via Switching Fuzzy Lyapunov Function Approach
}

\author{
Qunxian Zheng, Hongbin Zhang \\ School of Electronic Engineering, \\ University of Electronic Science and Technology of China, \\ Chengdu, Sichuan, 611731, P. R. China.
}

E-mail: Qunxian Zheng: zhengqx1103@163.com, Hongbin Zhang: zhanghb@uestc.edu.cn

\begin{abstract}
This paper addresses the problem of $H_{\infty}$ control for a class of continuous-time switched nonlinear systems via switching fuzzy Lyapunov functions (FLFs). The asynchronous switching is also considered in this paper, where "asynchronous" means that the switching of the controllers to be designed has a lag to the switching of the system models. The Takagi-Sugeno (T-S) fuzzy model is used here to approximate each nonlinear subsystem of the switched nonlinear systems. By using mode-dependent average dwell time (MDADT) techniques, we obtain stability conditions for the open-loop switched nonlinear systems and stabilization conditions with $H_{\infty}$ performance for the closed-loop switched nonlinear systems. The desired $H_{\infty}$ controller can be constructed by solving a set of linear matrix inequalities (LMIs). Finally, two numerical examples illustrate the utility and advantage of the proposed method.
\end{abstract}

Keyword: $H_{\infty}$ control, switched nonlinear systems, Takagi-Sugeno (T-S) fuzzy model, mode-dependent average dwell time (MDADT), switching fuzzy Lyapunov functions (FLFs). 


\section{Introduction}

Switched systems are an important class of hybrid systems. The last two decades have witnessed increasing research attention on switched systems, and many valuable stability and stabilization results have been obtained [1-5]. Up to now, two stability issues have been addressed in the literature, i.e., the stability under arbitrary switching and the stability under constrained switching. As one typical example of the constrained switching, the average dwell time (ADT) logic has been proposed in [6]. Stability problem and switching law design are addressed for switched systems with average dwell time [7-10]. However, most work assumed that the ADT is independent of the system models. By fully taking consideration of the characteristics of each subsystem, the mode-dependent average dwell time (MDADT) method is proposed in [11], which can further reduce the conservativeness of the results obtained by the ADT approach.

The considerations of the above work are focused on the switched linear systems. Recently, the control issue for the switched nonlinear systems has also been widely studied. Due to the nonlinearity, it is difficult to analyze the switched nonlinear systems directly. We used the well-known Takagi-Sugeno (T-S) fuzzy model to overcome such difficulties. The T-S fuzzy model is an effective tool in approximating most complex nonlinear systems [12], which utilizes local linear system description for each rule. The issue of stability and controller synthesis of T-S fuzzy systems has been studied extensively [13-20].

As we know that the results based on a common quadratic Lyapunov function (QLF) might be conservative. By taking into consideration the information of membership functions, the authors in $[21,22]$ presented a stability result for the continuous-time fuzzy system using fuzzy Lyapunov functions. The FLFs approach only needs to search for a local common positive matrix in fuzzy model and also reduces the difficulty of solving LMIs. In this paper, we investigate switched nonlinear systems by employing T-S fuzzy models. The switching FLFs are proposed by using the switching information and structural information of membership function in the rule base.

Recently, the $H_{\infty}$ control theory keeps attracting more and more attention because of its significant advances [23], and based on $H_{\infty}$ performance, a number of methodologies have been developed for the $H_{\infty}$ controller of filter design [24-30]. However, most of these 
results focused on the synchronous switching between the system model and the matched controller, which is a quite ideal case. In practice, due to system identification and other reasons, the matched controller of each subsystem would not be operated immediately at each switching instant. To design more practical controller, the work [7] and [10] have studied the asynchronous control problem of discrete-time and continuous-time switched linear systems, respectively. As far as we know, the problem of $H_{\infty}$ asynchronous control for continuoustime switched nonlinear systems remains to be unsolved, which motivates our research in this paper.

The main contributions of our work are as follows: (I) The previous work studied the $H_{\infty}$ asynchronous control problem of switched systems mainly focused on the switched linear systems. We use the T-S fuzzy model to study the $H_{\infty}$ asynchronous control problem of continuous-time switched nonlinear systems. Furthermore, our work can also reduce to study the $H_{\infty}$ asynchronous control problem of continuous-time switched linear systems. (II) In order to further relax the conservativeness resulted from the nonlinearity and the common Lyapunov functions approach, we propose the switching fuzzy Lyapunov functions approach, and this approach can also apply to study other switched nonlinear systems.

The rest of this paper is organized as follows. Section II gives preliminaries and problem formulation. The main results are given in Section III. In Section IV, a simulation example is presented. Finally, some conclusions are included in Section V.

Notations: The notation used in this paper is fairly standard. The symbol "*" in a matrix stands for the transposed elements in the symmetric positions. The superscript " $T$ " is the matrix transposition. $N$ and $N^{+}$denote the set of the natural numbers and the set of positive integers respectively. $R^{n}$ denotes the $n$-dimensional Euclidean space. $I$ and 0 represent the identity matrix and zero matrix respectively in the block matrix with appropriate dimensions. The notation $\|\cdot\|$ refers to the Euclidean vector norm. $L_{2}[0, \infty)$ is the space of squareintegrable and for $v(t) \in L_{2}[0, \infty)$, and its norm is given by $\|v(t)\|_{2}=\sqrt{\int_{0}^{\infty} v(t)^{T} v(t) d t}$. $C^{1}$ denotes the space of continuously differentiable function. A continuous function $\alpha:[0, \infty)$ $\rightarrow[0, \infty)$ is said to be of class $\mathcal{K}$ if it is strictly increasing and $\alpha(0)=0$. If $\alpha$ is also unbounded, then it is said to be of class $\mathcal{K}_{\infty}$. A function $\beta:[0, \infty) \times[0, \infty) \rightarrow[0, \infty)$ is said to be of class $\mathcal{K} \mathcal{L}$ if $\beta(\cdot, t)$ is of class $\mathcal{K}$ for each fixed $t \geq 0$ and $\beta(s, t)$ decreases to 0 
as $t \rightarrow \infty$ for each fixed $s \geq 0$. We use $P>0(\geq 0,<0, \leq 0)$ to denote a positive definite (semi-positive definite, negative definite, semi-negative definite) matrix $P$. If not explicitly stated, matrices are assumed to have compatible dimensions.

\section{Preliminaries and Problem Formulation}

Let us consider the following switched nonlinear systems described by

$$
\left\{\begin{array}{l}
\dot{x}(t)=f_{\sigma(t)}(x(t), u(t), w(t)) \\
y(t)=g_{\sigma(t)}(x(t), u(t), w(t))
\end{array}\right.
$$

where $x(t) \in R^{n_{x}}, u(t) \in R^{n_{u}}$, and $y(t) \in R^{n_{y}}$ denote the state vector, input vector and output vector respectively; $w(t) \in R^{n_{w}}$ is the disturbance that belongs to $L_{2}[0, \infty)$. $f_{\sigma(t)}$ and $g_{\sigma(t)}$ are nonlinear functions. $\sigma(t)$ is defined as a switching signal, which is a piecewise constant function of time and takes its values in the finite set $S=\{1,2, \ldots, N\}$, where $N$ is the number of subsystems. For a switching sequence $0<t_{0}<t_{1}<\cdots<t_{k}<t_{k+1}<\cdots, \sigma(t)$ is continuous from right everywhere. When $t \in\left[t_{k}, t_{k+1}\right)$, we say that the $\sigma\left(t_{k}\right)$ subsystem is activated, and for $\sigma\left(t_{k}\right)=i, i \in S$.

The T-S fuzzy model which is described by fuzzy IF-THEN rules [12] is employed here to represent each subsystem of the switched nonlinear systems.

Rule $n$ for subsystem $i$ : IF $\nu_{i 1}(t)$ is $N_{i 1 n}$ and $\cdots$ and $\nu_{i g}(t)$ is $N_{i g n}$, THEN

$$
\left\{\begin{array}{l}
\dot{x}(t)=A_{i n} x(t)+B_{i n} u(t)+E_{i n} w(t) \\
y(t)=C_{i n} x(t)+D_{i n} u(t)+F_{i n} w(t)
\end{array}\right.
$$

where $\nu_{i}(t)=\left(\nu_{i 1}(t), \nu_{i 2}(t), \cdots, \nu_{i g}(t)\right)$ are some measurable premise variables and $N_{i p n}(p=$ $1,2, \cdots, g)$ are fuzzy sets. $A_{i n}, B_{i n}, C_{i n}, D_{i n}, E_{i n}$ and $F_{i n}$ are constant real matrices of the $n$th local model of the $i$ th subsystem.

By using "fuzzy blending", the final output of the $i$ th subsystem is inferred as follows:

$$
\left\{\begin{array}{l}
\dot{x}(t)=\sum_{n=1}^{r_{i}} h_{i n}(t)\left[A_{i n} x(t)+B_{i n} u(t)+E_{i n} w(t)\right] \\
y(t)=\sum_{n=1}^{r_{i}} h_{i n}(t)\left[C_{i n} x(t)+D_{i n} u(t)+F_{i n} w(t)\right]
\end{array}\right.
$$

where $h_{i n}(t)=l_{i n}(t) / \sum_{n=1}^{r_{i}} l_{i n}(t), l_{i n}(t)=\prod_{p=1}^{g} N_{i p n}\left(\nu_{i p}(t)\right), r_{i}$ is the number of IF-THEN rules, and $N_{i p n}\left(\nu_{i p}(t)\right)$ is the grade of the membership function of $\nu_{i p}$ in $N_{i p n}$. It is assumed 
that $l_{\text {in }}(t) \geq 0$ for all $t$. Therefore, the normalized membership function $h_{i n}(t)$ satisfies

$$
h_{\text {in }}(t) \geq 0, \quad \sum_{n=1}^{r_{i}} h_{\text {in }}(t)=1 .
$$

In view of the asynchronous behaviors, the controller $u(t)$ is divided into two parts $\bar{u}(t)$ and $\hat{u}(t)$, where $\bar{u}(t)$ denotes the unmatched controller, and $\hat{u}(t)$ represents the matched controller. The terms $\bar{u}(t)$ and $\hat{u}(t)$ have the following form:

$$
\begin{cases}\bar{u}(t)=\sum_{m=1}^{r_{i}} h_{j m}(t) K_{j m} x(t), & t \in\left[t_{k}, \bar{t}_{k}\right) \\ \hat{u}(t)=\sum_{m=1}^{r_{i}} h_{i m}(t) K_{i m} x(t), & t \in\left[\bar{t}_{k}, t_{k+1}\right)\end{cases}
$$

where notation $\bar{t}_{k}\left(t_{k} \leq \bar{t}_{k}<t_{k+1}\right)$ denotes the starting-operating instant of the matched controller, and $K_{j m}$ and $K_{i m}$ are constant matrices. Substituting (5) into (3), we can obtain the following closed-loop system:

$$
\left\{\begin{array}{l}
\left\{\begin{array}{l}
\dot{x}(t)=\bar{A}_{i}(t) x(t)+E_{i}(t) w(t) \\
y(t)=\bar{C}_{i}(t) x(t)+F_{i}(t) w(t)
\end{array}, \quad \forall t \in\left[t_{k}, \bar{t}_{k}\right),\right. \\
\left\{\begin{array}{l}
\dot{x}(t)=\hat{A}_{i}(t) x(t)+E_{i}(t) w(t) \\
y(t)=\hat{C}_{i}(t) x(t)+F_{i}(t) w(t)
\end{array}, \quad \forall t \in\left[\bar{t}_{k}, t_{k+1}\right)\right.
\end{array}\right.
$$

where the $\bar{A}_{i}(t), \bar{C}_{i}(t), \hat{A}_{i}(t), \hat{C}_{i}(t), E_{i}(t)$ and $F_{i}(t)$ are defined as follows:

$$
\begin{array}{ll}
\bar{A}_{i}(t)=\sum_{n=1}^{r_{i}} \sum_{m=1}^{r_{i}} h_{i n}(t) h_{j m}(t)\left(A_{i n}+B_{i n} K_{j m}\right), & \bar{C}_{i}(t)=\sum_{n=1}^{r_{i}} \sum_{m_{i}=1}^{r_{i}} h_{i n}(t) h_{j m}(t)\left(C_{i n}+D_{i n} K_{j m}\right), \\
\hat{A}_{i}(t)=\sum_{n=1}^{r_{i}} \sum_{m=1}^{r_{i}} h_{i n}(t) h_{i m}(t)\left(A_{i n}+B_{i n} K_{i m}\right), & \hat{C}_{i}(t)=\sum_{n=1}^{r_{i}} \sum_{m=1}^{r_{i}} h_{i n}(t) h_{i m}(t)\left(C_{i n}+D_{i n} K_{i m}\right), \\
E_{i}(t)=\sum_{n=1}^{r_{i}} h_{i n}(t) E_{i n}, \quad F_{i}(t)=\sum_{n=1}^{r_{i}} h_{i n}(t) F_{i n} .
\end{array}
$$

To obtain the desired results, we introduce the following definitions.

Definition 1: [1] The switched system (1) with $w(t) \equiv 0$ is globally uniformly asymptotically stable (GUAS) if there exists a class $\mathcal{K} \mathcal{L}$ function $\beta$ such that for all switching signals $\sigma(t)$ and all initial condition $x\left(t_{0}\right)$, the solutions of (1) satisfy the following inequality

$$
\|x(t)\| \leq \beta\left(\left\|x\left(t_{0}\right)\right\|, t\right), \quad \forall t \geq t_{0} .
$$

Definition 2: [11] For switching signal $\sigma(t)$ and each $T \geq t \geq 0$. Let $N_{\sigma_{i}}(T, t)$ be the switching numbers such that the $i$ th subsystem is activated over the interval $[t, T]$, and $T_{i}(T, t)$ denotes the total running time of the $i$ th subsystem over the interval $[t, T], \forall i \in S$. 
We say that $\sigma(t)$ has a mode-dependent average dwell time (MDADT) $T_{a i}$ if there exist positive numbers $N_{0 i}\left(N_{0 i}\right.$ denotes mode-dependent chatter bounds here) and $T_{a i}$ such that

$$
N_{\sigma_{i}}(T, t) \leq N_{0 i}+T_{i}(T, t) / T_{a i}, \quad \forall T \geq t \geq 0
$$

Definition 3: [10] For $\alpha>0$ and $\gamma>0$, system (1) is said to have a weighted $L_{2}$-gain, if under zero initial condition (i.e., $x\left(t_{0}\right)=0$ ), it holds that

$$
\int_{0}^{\infty} e^{-\alpha s} y^{T}(s) y(s) d s \leq \gamma^{2} \int_{0}^{\infty} w^{T}(s) w(s) d s
$$

Remark 1: We can see from the above definition that if the system (1) has a $L_{2}$-gain, then it must have a weighted $L_{2}$-gain. However, the opposite is not true.

Definition 4: [21] Equation (10) is said to be a fuzzy Lyapunov function for the $i$ th subsystem of T-S fuzzy system (3) if there exists a positive definite matrix $P_{i m}$ and the time derivative of $V_{i}(x(t))$ is always negative at $x(t) \neq 0$.

$$
V_{i}(x(t))=x^{T}(t) P_{i}(t) x(t)
$$

where $P_{i}(t)=\sum_{m=1}^{r_{i}} h_{i m}(t) P_{i m}$ and $\dot{P}_{i}(t)=\sum_{m=1}^{r_{i}} \dot{h}_{i m}(t) P_{i m}$

\section{MAIN RESULTS}

In this section, for simplicity, let $T\left(t_{k}, t_{k+1}\right)=t_{k+1}-t_{k}$ denote the total running time of each subsystem in each interval. $T_{\uparrow}\left(t_{k}, t_{k+1}\right)$ denotes the running time of the unmatched controller in $\left[t_{k}, t_{k+1}\right)$, and the Lyapunov function may increase or decrease during $T_{\uparrow}\left(t_{k}, t_{k+1}\right)$. $T_{\downarrow}\left(t_{k}, t_{k+1}\right)$ represents the running time of the matched controller, and the Lyapunov function is strictly decreasing during $T_{\downarrow}\left(t_{k}, t_{k+1}\right)$.

\subsection{Stability analysis}

The open-loop system for (6) with $w(t) \equiv 0$ and $u(t) \equiv 0$ is listed as follow:

$$
\left\{\begin{array}{l}
\dot{x}(t)=\sum_{n=1}^{r_{i}} h_{i n}(t) A_{i n} x(t) \\
y(t)=\sum_{n=1}^{r_{i}} h_{i n}(t) C_{i n} x(t)
\end{array}, \quad \forall t \in\left[t_{k}, t_{k+1}\right) .\right.
$$

We present Lemma 1 which is useful for the stability analysis of the open-loop system (11). 
Lemma 1: Consider the system (11) and let $\alpha_{i}>0, \mu_{i} \geq 1$ be given constants. If there exist positive definite $C^{1}$ function $V_{\sigma\left(t_{k}\right)}: R^{n} \rightarrow R$, with $V_{\sigma\left(t_{0}\right)}\left(x_{t_{0}}\right) \equiv 0$ satisfying

$$
V_{i}\left(t_{k}\right) \leq \mu_{i} V_{j}\left(t_{k}^{-}\right)
$$

and

$$
\dot{V}_{i}(t) \leq-\alpha_{i} V_{i}(t), \quad t \in\left[t_{k}, t_{k+1}\right)
$$

Then the system (11) is GUAS for any switching signal satisfying

$$
T_{\text {oai }} \geq T_{\text {oai }}^{*}=\frac{\ln \mu_{i}}{\alpha_{i}} .
$$

Proof: By (13), we have

$$
V(t) \leq e^{-\alpha_{i}\left(t-t_{k}\right)} V\left(t_{k}\right)
$$

By (12) and (15), we have

$$
\begin{aligned}
V(t) & \leq \mu_{i} e^{-\alpha_{i}\left(t-t_{k}\right)} V\left(t_{k}^{-}\right) \\
& \leq \mu_{i} e^{-\alpha_{i}\left(t-t_{k}\right)-\alpha_{i-1}\left(t_{k}-t_{k-1}\right)} V\left(t_{k-1}\right) \\
& \leq \cdots \\
& \leq \mu_{i} \mu_{i-1} \cdots \mu_{1} e^{-\alpha_{i}\left(t-t_{k}\right)-\cdots-\alpha_{0}\left(t_{1}-t_{0}\right)} V\left(t_{0}\right) .
\end{aligned}
$$

Let $N_{\sigma i}$ denote $N_{\sigma i}\left(t, t_{0}\right)$ for simplicity. We get the following inequality

$$
V(t) \leq \exp \left\{\sum_{i=1}^{N} N_{\sigma i}\left(-\alpha_{i} T_{o a i}+\ln \mu_{i}\right)\right\} V\left(t_{0}\right)
$$

If supposing

$$
-\alpha_{i} T_{\text {oai }}+\ln \mu_{i} \leq 0,
$$

we obtain a sufficient condition that guarantees the GUAS of the system (11). The inequality (18) is equivalent to

$$
T_{\text {oai }} \geq T_{\text {oai }}^{*}=\frac{\ln \mu_{i}}{\alpha_{i}} .
$$

Therefore we can conclude that $V_{i}(t)$ converges to zero as $t \rightarrow \infty$ if the above condition is satisfied. This ends the proof.

The stability conditions for the system (11) can be summarized in the following theorem.

Theorem 1: Assume that

$$
\left|\dot{h}_{i s}(t)\right| \leq \varepsilon_{i s}, \quad s=1,2, \cdots, r_{i}
$$


where $\varepsilon_{i s} \geq 0$. Let $\alpha_{i}>0$ and $\mu_{i} \geq 1$ be given constants. The system (11) is GUAS for any switching signal satisfying (14), if there exist matrices $P_{i u}>0$ satisfying

$$
\begin{gathered}
P_{i u} \leq \mu_{i} P_{j v}, \quad P_{i k} \geq P_{i r_{i}}, \quad k=1,2, \cdots, r_{i}-1, \\
\Theta_{i n u}+\Theta_{i u n}<0, \quad n \leq u
\end{gathered}
$$

where

$$
\Theta_{i n u}=A_{i n}^{T} P_{i u}+P_{i u} A_{i n}+\sum_{s=1}^{r_{i}-1} \varepsilon_{i s}\left(P_{i s}-P_{i r_{i}}\right)+\alpha_{i} P_{i u} .
$$

Proof: Differentiating (4) implies

$$
\dot{h}_{i r_{i}}(t)=-\sum_{n=1}^{r_{i}-1} \dot{h}_{i n}(t),
$$

so we have

$$
\dot{P}_{i}(t)=\sum_{n=1}^{r_{i}-1} \dot{h}_{i n}(t)\left(P_{i n}-P_{i r_{i}}\right) .
$$

Combining (20), (21) with (24) implies

$$
\dot{P}_{i}(t) \leq \sum_{n=1}^{r_{i}-1} \varepsilon_{i n}\left(P_{i n}-P_{i r_{i}}\right) .
$$

Along the solution of the system (11), we have

$$
\begin{aligned}
\dot{V}_{i}(t)+\alpha_{i} V_{i}(t) & =x^{T}(t)\left[A_{i}^{T}(t) P_{i}(t)+P_{i}(t) A_{i}(t)+\dot{P}_{i}(t)+\alpha_{i} P_{i}(t)\right] x(t) \\
& \leq \sum_{n=1}^{r_{i}} h_{i n}^{2}(t) x^{T}(t) \Theta_{i n n} x(t)+\sum_{n<u}^{r_{i}} h_{i n}(t) h_{i u}(t) x^{T}(t)\left[\Theta_{i n u}+\Theta_{i u n}\right] x(t) .
\end{aligned}
$$

From (22) we can conclude that

$$
\dot{V}_{i}(t)+\alpha_{i} V_{i}(t) \leq 0 \text {. }
$$

For $P_{i u} \leq \mu_{i} P_{j v}$, we can get

$$
V_{i}\left(t_{k}\right) \leq \mu_{i} V_{j}\left(t_{k}^{-}\right)
$$

Combining (27) with (28) we conclude that the system (11) is GUAS for any switching signal satisfying (14). This completes the proof.

Remark 2: By setting $P_{i u}=P_{i}$, we can get the corresponding stability conditions based on the QLFs method. So the results in Theorem 1 might have less conservativeness than those results based on the QLFs method. 


\section{2 $H_{\infty}$ performance analysis and controller design}

Lemma 2: Consider the closed-loop switched nonlinear system (6), and let $\alpha_{i}>0, \beta_{i}>0$, $\gamma>0$ and $\mu_{i} \geq 1$ be given constants. If there exist positive definite $C^{1}$ function $V_{\sigma\left(t_{k}\right)}: R^{n}$ $\rightarrow R$, with $V_{\sigma\left(t_{0}\right)}\left(x_{t_{0}}\right) \equiv 0$ satisfying

$$
V_{i}\left(t_{k}\right) \leq \mu_{i} V_{j}\left(t_{k}^{-}\right)
$$

and

$$
\dot{V}_{i}(t) \leq\left\{\begin{array}{l}
\beta_{i} V_{i}(t)-J(t), \quad t \in T_{\uparrow}\left(t_{k}, t_{k+1}\right) \\
-\alpha_{i} V_{i}(t)-J(t), \quad t \in T_{\downarrow}\left(t_{k}, t_{k+1}\right)
\end{array}\right.
$$

where $J(t)=y^{T}(t) y(t)-\gamma^{2} w^{T}(t) w(t)$, then the system (6) is GUAS for any switching signal satisfying

$$
T_{c a i} \geq T_{c a i}^{*}=\frac{T_{M}\left(\beta_{i}+\alpha_{i}\right)+\ln \mu_{i}}{\alpha_{i}}
$$

and has a weighted $H_{\infty}$ performance no greater than $\hat{\gamma}=\sqrt{\exp \left\{\sum_{i=1}^{N}\left(T_{c a i}^{*} \alpha_{i}\right)+\theta_{\max } T_{M}\right\}} \gamma$, where $T_{M} \triangleq \max \left\{T_{\uparrow}\left(t_{k}, t_{k+1}\right), \quad \forall k=0,1,2, \ldots\right\}$.

Proof: Denote $\theta_{i}=\alpha_{i}+\beta_{i}, \theta_{\max }=\max \left\{\theta_{i}\right\}$ and $J(s)=y^{T}(s) y(s)-\gamma^{2} w^{T}(s) w(s)$. Then by (30), we have

$$
\begin{gathered}
V(t) \leq e^{-\alpha_{i}\left(t-t_{k}-T_{M}\right)+\beta_{i} T_{M}} V\left(t_{k}\right)-\int_{t_{k}+T_{M}}^{t} e^{-\alpha_{i}(t-s)} J(s) d s \\
-e^{-\alpha_{i}\left(t-t_{k}-T_{M}\right)} \int_{t_{k}}^{t_{k}+T_{M}} e^{\beta_{i}\left(t_{k}+T_{M}-s\right)} J(s) d s \\
=e^{-\alpha_{i}\left(t-t_{k}\right)+\theta_{i} T_{M}} V\left(t_{k}\right)-\int_{t_{k}+T_{M}}^{t} e^{-\alpha_{i}(t-s)} J(s) d s \\
\quad-\int_{t_{k}}^{t_{k}+T_{M}} e^{-\alpha_{i}(t-s)+\theta_{i}\left(t_{k}+T_{M}-s\right)} J(s) d s .
\end{gathered}
$$


By (29) and (32), we have

$$
\begin{aligned}
V(t) \leq & \mu_{i} e^{-\alpha_{i}\left(t-t_{k}\right)+\theta_{i} T_{M}} V\left(t_{k}^{-}\right)-\int_{t_{k}+T_{M}}^{t} e^{-\alpha_{i}(t-s)} J(s) d s \\
& -\int_{t_{k}}^{t_{k}+T_{M}} e^{-\alpha_{i}(t-s)+\theta_{i}\left(t_{k}+T_{M}-s\right)} J(s) d s \\
\leq & \mu_{i} e^{-\alpha_{i}\left(t-t_{k}\right)-\alpha_{i-1}\left(t_{k}-t_{k-1}\right)+\left(\theta_{i}+\theta_{i-1}\right) T_{M}} V\left(t_{k-1}\right) \\
& -\mu_{i} e^{-\alpha_{i}\left(t-t_{k}\right)+\theta_{i} T_{M}} \int_{t_{k-1}+T_{M}}^{t_{k}} e^{-\alpha_{i-1}\left(t_{k}-s\right)} J(s) d s \\
& -\mu_{i} e^{-\alpha_{i}\left(t-t_{k}\right)+\theta_{i} T_{M}} \int_{t_{k-1}}^{t_{k-1}+T_{M}} e^{-\alpha_{i-1}\left(t_{k}-s\right)+\theta_{i-1}\left(t_{k-1}+T_{M}-s\right)} J(s) d s \\
& -\int_{t_{k}}^{t_{k}+T_{M}} e^{-\alpha_{i}(t-s)+\theta_{i}\left(t_{k}+T_{M}-s\right)} J(s) d s-\int_{t_{k}+T_{M}}^{t} e^{-\alpha_{i}(t-s)} J(s) d s \\
\leq & \cdots \\
\leq & \mu_{i} \cdot \cdots \mu_{1} e^{-\alpha_{i}\left(t-t_{k}\right)-\cdots-\alpha_{0}\left(t_{1}-t_{0}\right)+T_{M}\left(\theta_{i}+\cdots+\theta_{0}\right)} V\left(t_{0}\right) \\
& -\mu_{i} \cdots \mu_{1} e^{-\alpha_{i}\left(t-t_{k}\right)-\cdots-\alpha_{1}\left(t_{2}-t_{1}\right)+T_{M}\left(\theta_{i}+\cdots+\theta_{1}\right)} \times\left[\int_{t_{0}+T_{M}}^{t_{1}} e^{-\alpha_{0}\left(t_{1}-s\right)} J(s) d s\right. \\
& \left.+\int_{t_{0}}^{t_{0}+T_{M}} e^{-\alpha_{0}\left(t_{1}-s\right)+\theta_{0}\left(t_{0}+T_{M}-s\right)} J(s) d s\right] \\
& \cdots-\int_{t_{k}}^{t_{k}+T_{M}} e^{-\alpha_{i}(t-s)+\theta_{i}\left(t_{k}+T_{M}-s\right)} J(s) d s-\int_{t_{k}+T_{M}}^{t} e^{-\alpha_{i}(t-s)} J(s) d s \\
= & (t)-\Omega_{(s)}(s)
\end{aligned}
$$

where

$$
\begin{aligned}
\Phi(t)= & e^{\ln \mu_{i}+\cdots+\ln \mu_{1}-\alpha_{i}\left(t-t_{k}\right)-\cdots-\alpha_{0}\left(t_{1}-t_{0}\right)+T_{M}\left(\theta_{i}+\cdots+\theta_{0}\right)} V\left(t_{0}\right) \\
= & \exp \left\{\sum_{i=1}^{N} N_{\sigma i}\left(-\alpha_{i} T_{c a i}+\ln \mu_{i}+\theta_{i} T_{M}\right)\right\} V\left(t_{0}\right) . \\
\Omega(s)= & \exp \left\{\sum_{i=1}^{N}\left(\ln \mu_{i}+\theta_{i} T_{M}\right)-\alpha_{i}\left(t-t_{k}\right)-\cdots-\alpha_{1}\left(t_{2}-t_{1}\right)\right\} \\
& \times\left[\int_{t_{0}+T_{M}}^{t_{1}} e^{-\alpha_{0}\left(t_{1}-s\right)} J(s) d s+\int_{t_{0}}^{t_{0}+T_{M}} e^{-\alpha_{0}\left(t_{1}-s\right)+\theta_{0}\left(t_{0}+T_{M}-s\right)} J(s) d s\right] \\
& +\cdots+\int_{t_{k}}^{t_{k}+T_{M}} e^{-\alpha_{i}(t-s)+\theta_{i}\left(t_{k}+T_{M}-s\right)} J(s) d s+\int_{t_{k}+T_{M}}^{t} e^{-\alpha_{i}(t-s)} J(s) d s .
\end{aligned}
$$

If supposing

$$
-\alpha_{i} T_{c a i}+\ln \mu_{i}+\theta_{i} T_{M} \leq 0,
$$

we obtain a sufficient condition that guarantees the GUAS of the switched nonlinear system 
(6). The inequality (34) can be rewritten as follows

$$
T_{c a i} \geq T_{c a i}^{*}=\frac{T_{M}\left(\beta_{i}+\alpha_{i}\right)+\ln \mu_{i}}{\alpha_{i}}
$$

Therefore we can conclude that $V_{i}(t) \rightarrow 0$ as $t \rightarrow \infty$ if (35) is satisfied.

We define $\bar{\Omega}(s)$ and $\hat{\Omega}(s)$ with the $y^{T}(s) y(s)$ and $\gamma^{2} w^{T}(s) w(s)$ terms, respectively. By defining $\alpha_{\max } \triangleq \max \left\{\alpha_{i}\right\}$ and letting all $\alpha_{i}=\alpha_{\max }$, we can obtain the following inequalities

$$
\Omega(s) \geq \bar{\Omega}(s)-\hat{\Omega}(s)
$$

where

$$
\begin{aligned}
\bar{\Omega}(s)= & \exp \left\{\sum_{i=1}^{N}\left(\ln \mu_{i}+\theta_{i} T_{M}\right)\right\} \times\left[\int_{t_{0}+T_{M}}^{t_{1}} e^{-\alpha_{\max }(t-s)} y^{T}(s) y(s) d s\right. \\
& \left.+\int_{t_{0}}^{t_{0}+T_{M}} e^{-\alpha_{\max }(t-s)+\theta_{0}\left(t_{0}+T_{M}-s\right)} y^{T}(s) y(s) d s\right] \\
& +\cdots \\
& +\int_{t_{k}}^{t_{k}+T_{M}} e^{-\alpha_{\max }(t-s)+\theta_{i}\left(t_{k}+T_{M}-s\right)} y^{T}(s) y(s) d s+\int_{t_{k}+T_{M}}^{t} e^{-\alpha_{\max }(t-s)} y^{T}(s) y(s) d s, \\
\hat{\Omega}(s)= & \exp \left\{\sum_{i=1}^{N}\left(\ln \mu_{i}+\theta_{i} T_{M}\right)\right\} \times\left[\int_{t_{0}+T_{M}}^{t_{1}} e^{-\alpha_{\max }(t-s)} \gamma^{2} w^{T}(s) w(s) d s\right. \\
& \left.+\int_{t_{0}}^{t_{0}+T_{M}} e^{-\alpha_{\max }(t-s)+\theta_{0}\left(t_{0}+T_{M}-s\right)} \gamma^{2} w^{T}(s) w(s) d s\right] \\
+ & \ldots \quad \int_{t_{k}}^{t_{k}+T_{M}} e^{-\alpha_{\max }(t-s)+\theta_{i}\left(t_{k}+T_{M}-s\right)} \gamma^{2} w^{T}(s) w(s) d s+\int_{t_{k}+T_{M}}^{t} e^{-\alpha_{\max }(t-s)} \gamma^{2} w^{T}(s) w(s) d s .
\end{aligned}
$$

Owing to $\exp \left\{\sum_{i=1}^{N}\left(\ln \mu_{i}+\theta_{i} T_{M}\right)\right\} \geq 1$ and $\theta_{i}\left(t_{k}+T_{M}-s\right) \geq 0$, we can have

$$
\begin{aligned}
\bar{\Omega}(s) \geq & \int_{t_{0}+T_{M}}^{t_{1}} e^{-\alpha_{\max }(t-s)} y^{T}(s) y(s) d s+\int_{t_{0}}^{t_{0}+T_{M}} e^{-\alpha_{\max }(t-s)} y^{T}(s) y(s) d s \\
& +\cdots+\int_{t_{k}}^{t_{k}+T_{M}} e^{-\alpha_{\max }(t-s)} y^{T}(s) y(s) d s+\int_{t_{k}+T_{M}}^{t} e^{-\alpha_{\max }(t-s)} y^{T}(s) y(s) d s \\
= & \int_{t_{0}}^{t} e^{-\alpha_{\max } t} y^{T}(s) y(s) d s
\end{aligned}
$$


Since $\theta_{\max } T_{M} \geq 0$ and $\theta_{i}\left(t_{k}+T_{M}-s\right) \leq \theta_{\max } T_{M}$, we can obtain the following inequality

$$
\begin{aligned}
& \hat{\Omega}(s) \leq \exp \left\{\sum_{i=1}^{N}\left(\ln \mu_{i}+\theta_{i} T_{M}\right)\right\} \times\left\{\int_{t_{0}+T_{M}}^{t_{1}} e^{-\alpha_{\max }(t-s)+\theta_{\max } T_{M}} \gamma^{2} w^{T}(s) w(s) d s\right. \\
&+\int_{t_{0}}^{t_{0}+T_{M}} e^{-\alpha_{\max }(t-s)+\theta_{\max } T_{M}} \gamma^{2} w^{T}(s) w(s) d s \\
&+\cdots+\int_{t_{k}}^{t_{k}+T_{M}} e^{-\alpha_{\max }(t-s)+\theta_{\max } T_{M}} \gamma^{2} w^{T}(s) w(s) d s \\
&\left.+\int_{t_{k}+T_{M}}^{t} e^{-\alpha_{\max }(t-s)+\theta_{\max } T_{M}} \gamma^{2} w^{T}(s) w(s) d s\right\} \\
&=\exp \left\{\sum_{i=1}^{N}\left(T_{c a i}^{*} \alpha_{i}\right)\right\} \times \int_{t_{0}}^{t} e^{-\alpha_{\max }(t-s)+\theta_{\max } T_{M}} \gamma^{2} w^{T}(s) w(s) d s .
\end{aligned}
$$

As for $\Omega(s)$, under zero initial condition, (33) gives

$$
\Omega(s) \leq 0
$$

Then, combining (36), (39), (40) with (41), the following inequality is obtained

$$
\int_{t_{0}}^{t} e^{-\alpha_{\max } t} y^{T}(s) y(s) d s \leq \hat{\gamma}^{2} \int_{t_{0}}^{t} e^{-\alpha_{\max }(t-s)} w^{T}(s) w(s) d s
$$

where $\hat{\gamma}=\sqrt{\exp \left\{\sum_{i=1}^{N}\left(T_{c a i}^{*} \alpha_{i}\right)+\theta_{\max } T_{M}\right\}} \gamma$. Let $t_{0}=0$, and integrating both sides of inequality (42) form $t=0$ to $\infty$ leads to

$$
\int_{0}^{\infty} e^{-\alpha_{\max } s} y^{T}(s) y(s) d s \leq \hat{\gamma}^{2} \int_{0}^{\infty} w^{T}(s) w(s) d s .
$$

This completes the proof.

Remark 3: In this proof, we consider the worst situation, that is the asynchronous time $T_{\uparrow}\left(t_{k}, t_{k+1}\right)(k=0,1,2, \cdots)$ takes its maximal value $T_{M}$ after every switching. Therefore, the results obtained in our work have more or less conservatism compared with the case that not all the asynchronous time $T_{\uparrow}\left(t_{k}, t_{k+1}\right)$ takes its maximal value $T_{M}$. In practice, if not all the asynchronous time $T_{\uparrow}\left(t_{k}, t_{k+1}\right)$ takes its maximal value $T_{M}$, the system can get GUAS with a relatively small $T_{c a i}^{*}$ and have a relatively better $H_{\infty}$ performance.

Remark 4: For the fuzzy Lyapunov function $V_{i}(x(t))=x^{T}(t) P_{i}(t) x(t)$, where $P_{i}(t)=$ $\sum_{m=1}^{r_{i}} h_{i m}(t) P_{i m}$ and $\dot{P}_{i}(t)=\sum_{m=1}^{r_{i}} \dot{h}_{i m}(t) P_{i m}$, we need to consider the time derivative of $h_{i m}(t)$, i.e., $\dot{h}_{i m}(t)$. Similar as in $[21,22]$, we replace the time derivative $\dot{h}_{i m}(t)$ with their upper bounds $\varepsilon_{i s}$. Furthermore, the reference [21] gave a method to obtain the time derivative 
upper bounds $\phi_{i m}$ in the condition that there is no external disturbance. As we know, compared with the system state $x(t)$, the disturbance $w(t)$ is relatively small and norm bounded, so we can obtain the time derivative upper bounds $\varepsilon_{i s}$ by adding a relatively small number to the $\phi_{i m}$ which is obtained by the method of [21].

Lemma 3: Let $\alpha_{i}>0, \beta_{i}>0, \gamma>0$ and $\mu_{i} \geq 1$ be given constants. The closed-loop switched nonlinear system (6) is GUAS for any switching signal satisfying (31) and has a weighted $H_{\infty}$ performance index $\hat{\gamma}$, if there exist matrices $P_{i}(t)>0$ satisfying

$$
\begin{gathered}
P_{i}(t) \leq \mu_{i} P_{j}(t), \\
{\left[\begin{array}{ccc}
\Xi_{i 1} & P_{i}(t) E_{i}(t) & \bar{C}_{i}^{T}(t) \\
* & -\gamma^{2} I & F_{i}^{T}(t) \\
* & * & -I
\end{array}\right]<0,} \\
{\left[\begin{array}{ccc}
\Xi_{i 2} & P_{i}(t) E_{i}(t) & \hat{C}_{i}^{T}(t) \\
* & -\gamma^{2} I & F_{i}^{T}(t) \\
* & * & -I
\end{array}\right]<0}
\end{gathered}
$$

where

$$
\begin{aligned}
& \Xi_{i 1}=P_{i}(t) \bar{A}_{i}(t)+\bar{A}_{i}^{T}(t) P_{i}(t)+\dot{P}_{i}(t)-\beta_{i} P_{i}(t), \\
& \Xi_{i 2}=P_{i}(t) \hat{A}_{i}(t)+\hat{A}_{i}^{T}(t) P_{i}(t)+\dot{P}_{i}(t)+\alpha_{i} P_{i}(t) .
\end{aligned}
$$

Proof: Supposing $i$ th subsystem is activated and the former one is $j$ th subsystem. By the system (6), considering $t \in T_{\uparrow}\left(t_{k}, t_{k+1}\right)$, from the Definition 4 , we have

$$
\dot{V}_{i}(t)-\beta_{i} V_{i}(t)+y^{T}(t) y(t)-\gamma^{2} w^{T}(t) w(t)=\eta^{T}(t)\left(\zeta_{i 1}+\zeta_{i 2}^{T} \zeta_{i 2}\right) \eta(t)
$$

where

$$
\eta^{T}(t)=\left[x^{T}(t), w^{T}(t)\right], \quad \zeta_{i 1}=\left[\begin{array}{cc}
\Xi_{i 1} & P_{i}(t) E_{i}(t) \\
* & -\gamma^{2} I
\end{array}\right], \quad \zeta_{i 2}=\left[\begin{array}{ll}
\bar{C}_{i}(t) & \left.F_{i}(t)\right] .
\end{array}\right.
$$

Similarly, for $t \in T_{\downarrow}\left(t_{k}, t_{k+1}\right)$, we obtain

$$
\dot{V}_{i}(t)+\alpha_{i} V_{i}(t)+y^{T}(t) y(t)-\gamma^{2} w^{T}(t) w(t)=\eta^{T}(t)\left(\zeta_{i 3}+\zeta_{i 4}^{T} \zeta_{i 4}\right) \eta(t)
$$

where

$$
\zeta_{i 3}=\left[\begin{array}{cc}
\Xi_{i 2} & P_{i}(t) E_{i}(t) \\
* & -\gamma^{2} I
\end{array}\right], \quad \zeta_{i 4}=\left[\begin{array}{ll}
\hat{C}_{i}(t) & F_{i}(t)
\end{array}\right]
$$


Using Schurs complement, we know that the inequalities (45) and (46) imply $\zeta_{i 1}+\zeta_{i 2}^{T} \zeta_{i 2}<0$ and $\zeta_{i 3}+\zeta_{i 4}^{T} \zeta_{i 4}<0$, respectively. By letting $J(s)=y^{T}(s) y(s)-\gamma^{2} w^{T}(s) w(s)$, we can obtain the following inequality:

$$
\dot{V}_{i}(t) \leq\left\{\begin{array}{l}
\beta_{i} V_{i}(t)-J(s), \quad t \in T_{\uparrow}\left(t_{k}, t_{k+1}\right) \\
-\alpha_{i} V_{i}(t)-J(s), \quad t \in T_{\downarrow}\left(t_{k}, t_{k+1}\right)
\end{array} .\right.
$$

Owing to $P_{i}(t) \leq \mu_{i} P_{j}(t)$, we can get

$$
V_{i}\left(t_{k}\right) \leq \mu_{i} V_{j}\left(t_{k}^{-}\right)
$$

According to Lemma 2, the system (6) is GUAS for any switching signal with MDADT satisfying (31) and has a weighted $H_{\infty}$ performance index $\hat{\gamma}$. The proof is ended.

In Lemma 3 the matrix variables $P_{i}(t)$ are coupled with the system parameter matrices in (45) and (46). Thus it is difficult to design the controller directly. We introduce the following lemma to solve this problem.

Lemma 4: Let $\alpha_{i}>0, \beta_{i}>0, \gamma>0$ and $\mu_{i} \geq 1$ be given constants. If there exist matrices $\tilde{P}_{i}(t)>0, L_{i}(t)$, and matrix $X$, satisfying

$$
\tilde{P}_{i}(t) \leq \mu_{i} \tilde{P}_{j}(t)
$$

and

where

$$
\begin{aligned}
& {\left[\begin{array}{cccc}
\Phi_{i 1} & \Phi_{i 2} & E_{i}(t) & \Phi_{i 3} \\
* & -X-X^{T} & E_{i}(t) & 0 \\
* & * & -\gamma^{2} I & F_{i}^{T}(t) \\
* & * & * & -I
\end{array}\right]<0,} \\
& {\left[\begin{array}{cccc}
\Psi_{i 1} & \Psi_{i 2} & E_{i}(t) & \Psi_{i 3} \\
* & -X-X^{T} & E_{i}(t) & 0 \\
* & * & -\gamma^{2} I & F_{i}^{T}(t) \\
* & * & * & -I
\end{array}\right]<0}
\end{aligned}
$$

$$
\begin{aligned}
& \Phi_{i 1}=A_{i}(t) X+X^{T} A_{i}^{T}(t)+B_{i}(t) L_{j}(t)+L_{j}^{T}(t) B_{i}^{T}(t)+\dot{\tilde{P}}_{i}(t)-\beta_{i} \tilde{P}_{i}(t), \\
& \Phi_{i 2}=\tilde{P}_{i}(t)-X+X^{T} A_{i}^{T}(t)+L_{j}(t) B_{i}^{T}(t), \quad \Phi_{i 3}=X^{T} C_{i}^{T}(t)+L_{j}^{T}(t) D_{i}^{T}(t), \\
& \Psi_{i 1}=A_{i}(t) X+X^{T} A_{i}^{T}(t)+B_{i}(t) L_{i}(t)+L_{i}^{T}(t) B_{i}^{T}(t)+\dot{\tilde{P}}_{i}(t)+\alpha_{i} \tilde{P}_{i}(t), \\
& \Psi_{i 2}=\tilde{P}_{i}(t)-X+X^{T} A_{i}^{T}(t)+L_{i}(t) B_{i}^{T}(t), \quad \Psi_{i 3}=X^{T} C_{i}^{T}(t)+L_{i}^{T}(t) D_{i}^{T}(t) .
\end{aligned}
$$


Then, we can conclude that the inequalities (44), (45) and (46) hold.

Proof: In order to decouple the matrix variables $P_{i}(t)$ and system parameter matrices, we introduce a slack matrix $Q$. By introducing a slack matrix $Q$, we now introducing the following inequalities:

$$
\begin{gathered}
{\left[\begin{array}{cccc}
\xi_{i 1} & P_{i}(t)-Q^{T}+\bar{A}_{i}^{T}(t) Q & Q^{T} E_{i}(t) & \bar{C}_{i}^{T}(t) \\
* & -Q-Q^{T} & Q^{T} E_{i}(t) & 0 \\
* & * & -\gamma^{2} I & F_{i}^{T}(t) \\
* & * & * & -I
\end{array}\right]<0,} \\
{\left[\begin{array}{cccc}
\xi_{i 2} & P_{i}(t)-Q^{T}+\hat{A}_{i}^{T}(t) Q & Q^{T} E_{i}(t) & \hat{C}_{i}^{T}(t) \\
* & -Q-Q^{T} & Q^{T} E_{i}(t) & 0 \\
* & * & -\gamma^{2} I & F_{i}^{T}(t) \\
* & * & * & -I
\end{array}\right]<0}
\end{gathered}
$$

where

$$
\xi_{i 1}=Q^{T} \bar{A}_{i}(t)+\bar{A}_{i}^{T}(t) Q+\dot{P}_{i}(t)-\beta_{i} P_{i}(t), \quad \xi_{i 2}=Q^{T} \hat{A}_{i}(t)+\hat{A}_{i}^{T}(t) Q+\dot{P}_{i}(t)+\alpha_{i} P_{i}(t) .
$$

Multiplying (54) from the left and right, respectively, by $\bar{\Lambda}_{i}(t)$ and its transpose, and multiplying (55) from the left and right, respectively, by $\hat{\Lambda}_{i}(t)$ and its transpose, where

$$
\bar{\Lambda}_{i}=\left[\begin{array}{cccc}
I & \bar{A}_{i}^{T}(t) & 0 & 0 \\
0 & E_{i}^{T}(t) & I & 0 \\
0 & 0 & 0 & I
\end{array}\right], \quad \hat{\Lambda}_{i}=\left[\begin{array}{cccc}
I & \hat{A}_{i}^{T}(t) & 0 & 0 \\
0 & E_{i}^{T}(t) & I & 0 \\
0 & 0 & 0 & I
\end{array}\right] .
$$

Then we conclude that (45) and (46) hold. Notice that if the conditions in (54) and (55) hold, the matrix $Q$ is nonsingular. We define the following matrices:

$$
X=Q^{-1}, \quad L_{i}(t)=K_{i}(t) X, \quad \tilde{P}_{i}(t)=X^{T} P_{i}(t) X
$$

Multiplying (54) from the left and right by diagonal matrix $\operatorname{diag}\left(Q^{-T}, Q^{-T}, I, I\right)$ and its transpose respectively. We can obtain

$$
\left[\begin{array}{cccc}
\xi_{i 3} & \xi_{i 4} & E_{i}(t) & Q^{-T} \bar{C}_{i}^{T}(t) \\
* & -Q^{-1}-Q^{-T} & E_{i}(t) & 0 \\
* & * & -\gamma^{2} I & F_{i}^{T}(t) \\
* & * & * & -I
\end{array}\right]<0
$$


where

$$
\begin{aligned}
& \xi_{i 3}=\bar{A}_{i}(t) Q^{-1}+Q^{-T} \bar{A}_{i}^{T}(t)+Q^{-T} \dot{P}_{i}(t) Q^{-1}-\beta_{i} Q^{-T} P_{i}(t) Q^{-1}, \\
& \xi_{i 4}=Q^{-T} P_{i}(t) Q^{-1}-Q^{-1}+Q^{-T} \bar{A}_{i}^{T}(t) .
\end{aligned}
$$

By the similar operation to (55), we can get

$$
\left[\begin{array}{cccc}
\xi_{i 5} & \xi_{i 6} & E_{i}(t) & Q^{-T} \hat{C}_{i}^{T}(t) \\
* & -Q^{-1}-Q^{-T} & E_{i}(t) & 0 \\
* & * & -\gamma^{2} I & F_{i}^{T}(t) \\
* & * & * & -I
\end{array}\right]<0
$$

where

$$
\begin{aligned}
& \xi_{i 5}=\hat{A}_{i}(t) Q^{-1}+Q^{-T} \hat{A}_{i}^{T}(t)+Q^{-T} \dot{P}_{i}(t) Q^{-1}+\alpha_{i} Q^{-T} P_{i}(t) Q^{-1}, \\
& \xi_{i 6}=Q^{-T} P_{i}(t) Q^{-1}-Q^{-1}+Q^{-T} \hat{A}_{i}^{T}(t) .
\end{aligned}
$$

By considering (54)-(56) and the definition of matrices $\bar{A}_{i}(t), \bar{C}_{i}(t), \hat{A}_{i}(t)$ and $\hat{C}_{i}(t)$ in $(6)$, we conclude that LMIs (57) and (58) imply (52) and (53) respectively. Moreover, by performing a congruence transformation to $P_{i}(t) \leq \mu_{i} P_{j}(t)$, we obtain

$$
Q^{-T} P_{i}(t) Q^{-1} \leq \mu_{i} Q^{-T} P_{j}(t) Q^{-1} \Leftrightarrow \tilde{P}_{i}(t) \leq \mu_{i} \tilde{P}_{j}(t)
$$

Thus, if (51)-(53) hold, we can conclude that (44)-(46) hold. This completes the proof.

Remark 5: Due to the existence of $\dot{h}_{i m}(t)$, the conditions in lemma 2 and lemma 3 can not be solved analytically or even numerically. Similar as [21,22], we transform the conditions of lemma 2 and lemma 3 into the conditions which can be solved numerically by replacing the time derivative $\dot{h}_{i m}(t)$ with their upper bounds $\varepsilon_{i m}$. Then, a reasonable estimation of $\dot{P}_{i}(t)$ is give as $\dot{P}_{i}(t)=\sum_{m=1}^{r_{i}} \dot{h}_{i m}(t) P_{i m} \leq \sum_{m=1}^{r_{i}} \varepsilon_{i m} P_{i m}$.

The following theorem provides the stabilization conditions with $H_{\infty}$ performance for the closed-loop switched nonlinear system (6).

Theorem 2: Assume that

$$
\left|\dot{h}_{i s}(t)\right| \leq \varepsilon_{i s}, \quad s=1,2, \cdots, r_{i}
$$

where $\varepsilon_{i s} \geq 0$. Let $\alpha_{i}>0, \beta_{i}>0, \gamma>0$ and $\mu_{i} \geq 1$ be given constants. If there exist matrices $\tilde{P}_{i u}>0, L_{i n}$ and matrix $X$ satisfying

$$
\tilde{P}_{i u} \leq \mu_{i} \tilde{P}_{j v}, \quad \tilde{P}_{i k} \geq \tilde{P}_{i r_{i}}, \quad k=1,2, \cdots, r_{i}-1,
$$


and

$$
\left\{\begin{array}{l}
\bar{\Theta}_{i m n u}+\bar{\Theta}_{i u n m}<0, \quad n \leq u \\
\hat{S}_{i m m m}=\hat{\Theta}_{i m m m}<0, \\
\hat{S}_{i m m n}=\frac{1}{3}\left[\hat{\Theta}_{i m m n}+\hat{\Theta}_{i m n m}+\hat{\Theta}_{i n m m}\right]<0, m \neq n, \\
\hat{S}_{i m n u}=\frac{1}{6}\left[\hat{\Theta}_{i m n u}+\hat{\Theta}_{i m u n}+\hat{\Theta}_{i n m u}+\hat{\Theta}_{i n u m}+\hat{\Theta}_{i u n m}+\hat{\Theta}_{i u m n}\right]<0, m<n<u
\end{array}\right.
$$

for all $m, n, u, v \in\left\{1,2, \ldots, r_{i}\right\}$, where

$$
\bar{\Theta}_{i m n u}=\left[\begin{array}{cccc}
\Phi_{i 1} & \Phi_{i 2} & E_{i n} & \Phi_{i 3} \\
* & -X-X^{T} & E_{i n} & 0 \\
* & * & -\gamma^{2} I & F_{i n}^{T} \\
* & * & * & -I
\end{array}\right], \hat{\Theta}_{i m n u}=\left[\begin{array}{cccc}
\Psi_{i 1} & \Psi_{i 2} & E_{i n} & \Psi_{i 3} \\
* & -X-X^{T} & E_{i n} & 0 \\
* & * & -\gamma^{2} I & F_{i n}^{T} \\
* & * & * & -I
\end{array}\right],
$$

and

$$
\begin{aligned}
& \Phi_{i 1}=A_{i n} X+X^{T} A_{i n}^{T}+B_{i n} L_{j m}+L_{j m}^{T} B_{i n}^{T}+\sum_{s=1}^{r_{i}-1} \varepsilon_{i s}\left(\tilde{P}_{i s}-\tilde{P}_{i r_{i}}\right)-\beta_{i} \tilde{P}_{i u}, \\
& \Phi_{i 2}=\tilde{P}_{i u}-X+X^{T} A_{i n}^{T}+L_{j m}^{T} B_{i n}^{T}, \quad \Phi_{i 3}=X^{T} C_{i n}^{T}+L_{j m}^{T} D_{i n}^{T}, \\
& \Psi_{i 1}=A_{i n} X+X^{T} A_{i n}^{T}+B_{i n} L_{i m}+L_{i m}^{T} B_{i n}^{T}+\sum_{s=1}^{r_{i}} \varepsilon_{i s}\left(\tilde{P}_{i s}-\tilde{P}_{i r_{i}}\right)+\alpha_{i} \tilde{P}_{i u}, \\
& \Psi_{i 2}=\tilde{P}_{i u}-X+X^{T} A_{i n}^{T}+L_{i m}^{T} B_{i n}^{T}, \quad \Psi_{i 3}=X^{T} C_{i n}^{T}+L_{i m}^{T} D_{i n}^{T},
\end{aligned}
$$

then the system (6) is GUAS for any switching signal with MDADT satisfying (31) and has a weighted $H_{\infty}$ performance index $\hat{\gamma}$. Moreover, if a feasible solution exists, the admissible controller gains can be given by

$$
\begin{cases}K_{j m}=L_{j m} X^{-1}, & t \in\left[t_{k}, \bar{t}_{k}\right) \\ K_{i m}=L_{i m} X^{-1}, & t \in\left[\bar{t}_{k}, t_{k+1}\right) .\end{cases}
$$

Proof: Denote the left side of $(52)$ and $(53)$ as $\bar{\Theta}_{i}(t)$ and $\hat{\Theta}_{i}(t)$, respectively. If the conditions in Theorem 2 are satisfied, we can obtain

$$
\begin{aligned}
\bar{\Theta}_{i}(t) & \leq \sum_{m=1}^{r_{i}} \sum_{n=1}^{r_{i}} \sum_{u=1}^{r_{i}} h_{i n}(t) h_{j m}(t) h_{i u}(t) \bar{\Theta}_{i m n u} \\
& =\sum_{m=1}^{r_{i}} h_{j m}(t)\left[\sum_{n=1}^{r_{i}} h_{i n}^{2}(t) \bar{\Theta}_{i n m n}+\sum_{n=1}^{r_{i}} \sum_{n<u}^{r_{i}} h_{i n}(t) h_{i u}(t)\left(\bar{\Theta}_{i n m u}+\bar{\Theta}_{i u m n}\right)\right]<0
\end{aligned}
$$

and

$$
\begin{aligned}
\hat{\Theta}_{i}(t) \leq & \sum_{m=1}^{r_{i}} \sum_{n=1}^{r_{i}} \sum_{u=1}^{r_{i}} h_{i m}(t) h_{i n}(t) h_{i u}(t) \hat{\Theta}_{i m n u} \\
= & \sum_{m=1}^{r_{i}} h_{i m}^{3}(t) \hat{S}_{i m m m}+3 \sum_{m=1}^{r_{i}} \sum_{\substack{n=1 \\
n \neq m}}^{r_{i}} h_{i m}(t) h_{i n}(t) \hat{S}_{i m m n} \\
& +6 \sum_{m=1}^{r_{i}} \sum_{n>m} \sum_{u>n} h_{i m}(t) h_{i n}(t) h_{i u}(t) \hat{S}_{i m n u}<0 .
\end{aligned}
$$


From Lemmas 2, 3 and 4, we know the $H_{\infty}$ controller design problem is solved. By (56), we can conclude that the gains in (5) can be constructed by (64). This completes the proof.

Remark 6: Theorem 2 can also be applicable to relevant problems for the switched nonlinear system without asynchronous behaviors. For this case, if (62) in Theorem 2 is removed, we can conclude that the system is GUAS and has a weighted $H_{\infty}$ performance index $\hat{\gamma}_{s}=\sqrt{\exp \left\{\sum_{i=1}^{N}\left(T_{a i}^{*} \alpha_{i}\right)\right\}} \gamma$ for any switching signal satisfies $T_{a i} \geq T_{a i}^{*}=\frac{\ln \mu_{i}}{\alpha_{i}}$.

Remark 7: All the parameters $\left(\alpha_{i}, \beta_{i}, \mu_{i}\right.$ and $\left.\gamma\right)$ of Theorem 2 have their physical meaning, and these parameters can be designed according to the system performance requirements. For example, the parameter $\alpha_{i}$ denotes the decline rate of the Lyapunov function, which corresponds to the convergence rate of the system in synchronous state. And the parameter $\beta_{i}$ denotes the increasing rate of the Lyapunov function when the system is running in the asynchronous state. In practice, we can design the values of these parameter to make the systems reach the desired performance.

Remark 8: In our method, in order to reduce the fuzzy approximation error, much more fuzzy rules should be used, which will cause to greater computational complexity.

\section{Numerical Example}

In this section, two numerical examples are given to illustrate the effectiveness and advantage of our results.

Example 1: Let $x(t)=\left[\begin{array}{ll}x_{1}^{T}(t) & x_{2}^{T}(t)\end{array}\right]^{T}$, and consider the open-loop switched fuzzy system (11) consisting of two subsystems, and each subsystem has two fuzzy rules, where

$$
A_{11}=\left[\begin{array}{cc}
a & -4 \\
-1 & -2
\end{array}\right], \quad A_{12}=\left[\begin{array}{ll}
-2 & -4 \\
20 & -2
\end{array}\right], A_{21}=\left[\begin{array}{cc}
-5 & -2 \\
-6 & -3
\end{array}\right], \quad A_{22}=\left[\begin{array}{cc}
b & -2 \\
15 & -3
\end{array}\right] .
$$

The fuzzy membership functions are taken as

$$
\begin{aligned}
& h_{11}=1-\frac{2}{1+\exp \left(-x_{1}\right)} \times \frac{2}{1+\exp \left(x_{1}\right)}, \quad h_{12}=1-h_{11}, \\
& h_{21}=\sin ^{2}\left(x_{2}+0.5\right), \quad h_{22}=1-h_{21} .
\end{aligned}
$$

By the method of [21], we can obtain the parameters $\varepsilon_{i s}$ as $\varepsilon_{11}=\varepsilon_{12}=1$ and $\varepsilon_{21}=\varepsilon_{22}=0.5$.

In order to show the merit of the FLFs method, we compare the feasible area for the stability conditions based on the FLFs method and QLFs method by changing $a$ and $b$. 
The parameters used in these two methods have the same values, that is $\mu_{1}=2, \mu_{2}=2$, $\alpha_{1}=0.1$ and $\alpha_{2}=0.15$. The simulation results are shown in Figure 1. It can be seen from Figure 1 that the feasible area for FLFs method is larger than that for QLFs method, so the conditions of QLFs method lead to conservative results compared with FLFs method.

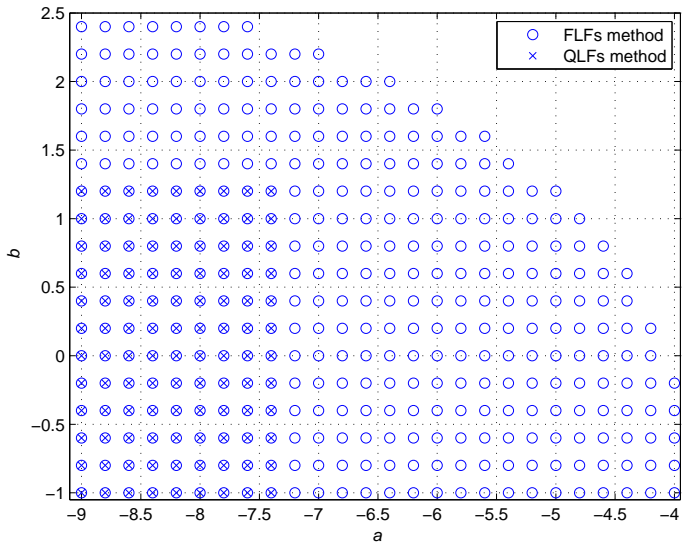

Figure 1: Feasible area for the stability conditions of FLFs method and QLFs method 1, where $a$ and $b$ take a step of 0.2 .

Example 2: Consider the closed-loop switched fuzzy system (6) consisting of two subsystems, and each subsystem has two fuzzy rules, where

$$
\begin{aligned}
& A_{11}=\left[\begin{array}{cc}
-0.15 & -0.03 \\
0 & 0.15
\end{array}\right], A_{12}=\left[\begin{array}{cc}
-0.1 & -0.05 \\
0 & 0.1
\end{array}\right], A_{21}=\left[\begin{array}{cc}
0.1 & 0 \\
0.15 & -0.05
\end{array}\right], \\
& A_{22}=\left[\begin{array}{ll}
0.4 & 0 \\
0.1 & -0.15
\end{array}\right], B_{11}=\left[\begin{array}{cc}
0.25 & -0.08 \\
0.1 & 0.16
\end{array}\right], B_{12}=\left[\begin{array}{cc}
0.2 & 0.01 \\
0.1 & 0.15
\end{array}\right], \\
& B_{21}=\left[\begin{array}{ll}
0.65 & -0.01 \\
0.02 & -0.5
\end{array}\right], \quad B_{22}=\left[\begin{array}{cc}
0.58 & -0.01 \\
0.01 & -0.4
\end{array}\right], \\
& C_{11}=\left[\begin{array}{ll}
0.3 & 0.5
\end{array}\right], C_{12}=\left[\begin{array}{ll}
0.2 & 0.2
\end{array}\right], C_{21}=\left[\begin{array}{l}
0.15 \\
0.25
\end{array}\right], C_{22}=\left[\begin{array}{ll}
0.2 & 0.2
\end{array}\right], \\
& D_{11}=\left[\begin{array}{ll}
0.25 & 0.25
\end{array}\right], D_{12}=\left[\begin{array}{ll}
0.25 & 0.25
\end{array}\right], D_{21}=\left[\begin{array}{ll}
0.2 & 0.2
\end{array}\right], D_{22}=\left[\begin{array}{ll}
0.18 & 0.22
\end{array}\right], \\
& E_{11}=\left[\begin{array}{l}
0.35 \\
0.22
\end{array}\right], E_{12}=\left[\begin{array}{l}
0.1 \\
0.1
\end{array}\right], E_{21}=\left[\begin{array}{l}
0.1 \\
0.1
\end{array}\right], E_{22}=\left[\begin{array}{l}
0.12 \\
0.08
\end{array}\right], \\
& F_{11}=0.1, \quad F_{12}=0.05, \quad F_{21}=0.1, \quad F_{22}=0.1 .
\end{aligned}
$$

The fuzzy membership functions used here are the same as in Example 1. The initial 
conditions are assumed to be $x\left(t_{0}\right)=[1,1.5]^{T}$, and the disturbance input is assumed to be $w(t)=\sin (\pi t) \exp (-0.15 t)$. We choose $\gamma=2, N_{0 i}=0, T_{M}=1, \mu_{1}=1.1, \mu_{2}=1.2$, $\alpha_{1}=0.15, \alpha_{2}=0.2, \beta_{1}=0.05$ and $\beta_{2}=0.1$. So by Theorem 2 , we obtain $T_{c a 1}^{*}=1.9687$, $T_{c a 2}^{*}=2.4116$ and $\hat{\gamma}=3.4280$.

It is clear that the uncontrolled system is unstable. We will use our results to design a set of mode-dependent controllers such that the closed-loop switched system (6) is GUAS and the $H_{\infty}$ performance is achieved. In practice, the asynchronous switching between the systems and the controllers generally exists. First, we study the asynchronous switching, while the controllers are designed by only considering the synchronous conditions. By solving (61) and (63) with the above parameters, the controller gains can be obtained as follows:

$$
\begin{aligned}
& K_{11}=\left[\begin{array}{cc}
-1.4709 & 1.0249 \\
0.4338 & -3.5225
\end{array}\right], K_{12}=\left[\begin{array}{cc}
-1.5182 & 2.2835 \\
0.7339 & -3.6958
\end{array}\right], \\
& K_{21}=\left[\begin{array}{ll}
-0.8744 & -1.0862 \\
-0.2575 & 1.6865
\end{array}\right], K_{22}=\left[\begin{array}{cc}
-1.4171 & -0.7935 \\
-0.1907 & 1.4885
\end{array}\right] .
\end{aligned}
$$

The state response for this case is shown in Figure 2. As shown in Figure 2 the systems become unstable if we ignore the effects of asynchronous behavior on the controllers design.

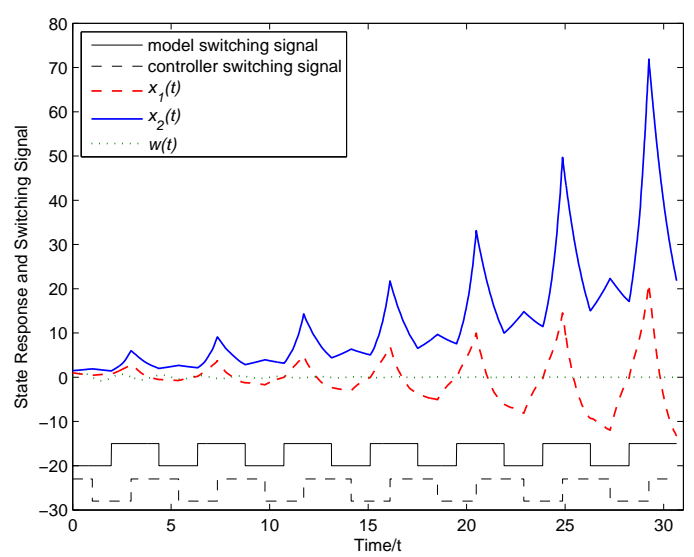

Figure 2: State response of the closed-loop asynchronous switched systems with controllers designed by synchronous conditions.

Then, we investigate the asynchronous switching and the controllers are designed by considering the synchronous and asynchronous conditions. By solving (61), (62) and (63) 
with the above parameters, the controller gains are listed as follows:

$$
\begin{aligned}
& K_{11}=\left[\begin{array}{cc}
0.7701 & -18.5215 \\
-0.0831 & 3.4435
\end{array}\right], K_{12}=\left[\begin{array}{cc}
1.1679 & -19.0306 \\
-0.2474 & 4.3554
\end{array}\right], \\
& K_{21}=\left[\begin{array}{cc}
1.2707 & -19.9038 \\
-0.1819 & 4.4981
\end{array}\right], K_{22}=\left[\begin{array}{cc}
0.9029 & -16.9572 \\
-0.2061 & 3.6144
\end{array}\right] .
\end{aligned}
$$

The state response for this situation is shown in Figure 3. As is shown in Figure 3 that the states of the closed-loop system converge to zero. It means that the designed controllers by considering asynchronous conditions are effective. Since the asynchronous switching is very common in real world, it is necessary for us to consider the effects of asynchronous behavior on the controllers design. Through comparing Figure 2 with Figure 3, we can conclude if we ignore the effects of asynchronous behavior on the controllers design, the controllers designed can not meet the actual requirements.

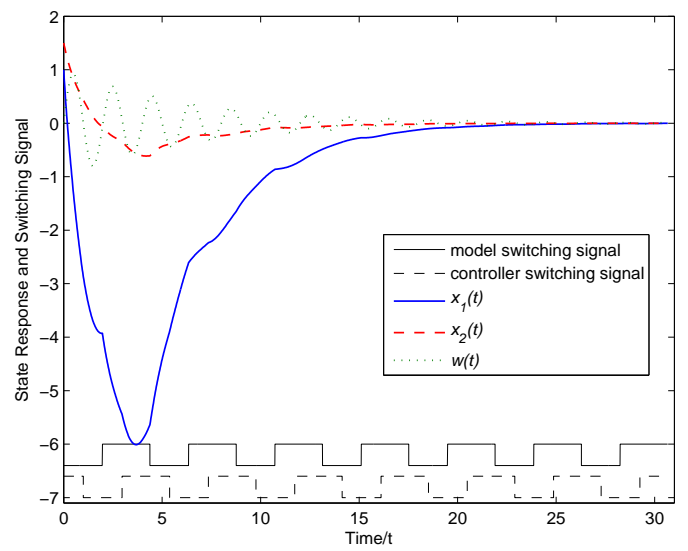

Figure 3: State response of the closed-loop asynchronous switched systems with controllers designed by synchronous and asynchronous conditions.

\section{Conclusion}

In this study, the switching FLFs are used to investigate the $H_{\infty}$ control problem for the switched nonlinear systems with asynchronous behaviors. By using MDADT techniques, we obtain stability conditions for the open-loop switched nonlinear systems and stabilization conditions with $H_{\infty}$ performance for the closed-loop switched nonlinear systems. A 
state-feedback fuzzy controller is designed, which can ensure the stability of the closed-loop switched system with a weighted $H_{\infty}$ performance. We remark that the obtained results can also apply to the switched nonlinear system without asynchronous behaviors. Finally, two numerical examples are given to demonstrate the effectiveness and advantage of our method. The obtained results might be used in the controller design for the network control system. Then potential extensions of our current results to the stability analysis and control synthesis for the systems under the network-based environment [31] or Markovian jump systems with time delay [32] need further study, which will be our future work. On the other hand, it has been shown that the piecewise Lyapunov functions method is another way to relax the conservativeness inherent in common Lyapunov function method [25,26], and a T-S fuzzy affine dynamic model has much improved function approximation capabilities $[25,26]$. Therefore it would be our another future work to investigate the $H_{\infty}$ control problem for the T-S fuzzy affine systems via the piecewise Lyapunov functions method.

\section{Acknowledgement}

The work was supported in part by the National Natural Science Foundation of China (Grant no. 61374117, Grant no, 61004048, Grant no. 61174137, Grant no. 61104038 and Grant no. 61374086), the NSF of Jiang Su Province (Grant no. BK2010493), the grant from China Postdoctoral Science Foundation funded project 2012M510135, the Program for Changjiang Scholars and Innovative Research Team in University, the project form science \& technology department of Sichuan province(Grant no. 2013GZ0080), the 973 project 2011CB707000.

\section{References}

[1] D. Liberzon, Switching in systems and control, Berlin, Germany: Birkhauser, 2003.

[2] H. Ye, A.N. Michel, L. Hou, Stability theory for hybrid dynamical systems, IEEE Trans. Autom. Control 43 (4) (1998) 461-474.

[3] S.H. Lee, T.H. Kim, J.T. Lim, A new stability analysis of switched systems, Automatica 36 (6) (2000) 917-922. 
[4] D. Cheng, L. Guo, Y. Lin, Y. Wang, Stabilization of switched linear systems, IEEE Trans. Autom. Control 50 (5) (2005) 661-666.

[5] D. Liberzon, A.S. Morse, Basic problems in stability and design of switched systems, IEEE Control Syst. Mag. 19 (5) (1999) 59-70.

[6] J.P. Hespanha, A.S. Morse, Stability of switched systems with average dwell-time. in: Proc. IEEE Conf. Decision and Control, Phoenix, AZ, 1999, pp. 2655-2660.

[7] L. Zhang, P. Shi, Stability, $l_{2}$-gain and asynchronous $H_{\infty}$ control of discrete-time switched systems with average dwell time, IEEE Trans. Autom. Control 54 (9) (2009) 2193-2200.

[8] L. Zhang, H. Gao, Asynchronously switched control of switched systems with average dwell time, Automatica 46 (5) (2010) 953-958.

[9] L. Zhang, N. Cui, M. Liu, Y. Zhao, Asynchronous filtering of discrete-time switched linear systems with average dwell time, IEEE Trans. Circuits Syst. 58 (5) (2011) 11091118.

[10] H. Zhang, B. Wang, D. Xie, S. Xu, C. Dang, Stability, $L_{2}$-gain and asynchronous $H_{\infty}$ control for continuous-time switched systems, Int. J. Robust Nonlinear Control 25 (4) (2015) 575-587.

[11] X. Zhao, L. Zhang, P. Shi, M. Liu, Stability and stabilization of switched linear systems with mode-dependent average dwell time, IEEE Trans. Autom. Control 57 (7) (2012) 1809-1815.

[12] T. Takagi, M. Sugeno, Fuzzy identification of systems and its application to modeling and control, IEEE Trans. Syst., Man, Cybern. SMC-15 (1) (1985) 116-132.

[13] K. Tanaka, H.O. Wang, Fuzzy control systems design and analysis: A linear matrix inequality approach, New York: Wiley, 2001.

[14] K. Tanaka, T. Ikeda, H.O. Wang, Fuzzy regulator and fuzzy observer: Relaxed stability conditions and LMI-based designs, IEEE Trans. Fuzzy Syst. 6 (2) (1998) 250-265. 
[15] K. Tanaka, M. Sugeno, Stability analysis and design of fuzzy control systems, Fuzzy Sets Syst. 45 (2) (1992) 135-156.

[16] J. Qiu, H. Tian, Q. Lu, H. Gao, Nonsynchronized robust filtering design for continuoustime T-S fuzzy affine dynamic systems based on piecewise Lyapunov functions, IEEE Trans. Cybern. 43 (6) (2013) 1755-1766.

[17] Y. Mao, H. Zhang, S. Xu, Exponential stability and asynchronous stabilization of a class of switched nonlinear system via T-S fuzzy model, IEEE Trans. Fuzzy Syst. 22 (4) (2014) 817-828.

[18] Y. Hou, S. Tong, Adaptive fuzzy output-feedback control for a class of nonlinear switched systems with unmodeled dynamics, Neurocomputing 168 (30) (2015) 200-209.

[19] H. Li, Y. Gao, L. Wu, H.K. Lam, Fault detection for T-S fuzzy time-delay systems: delta operator and input-output methods, IEEE Trans. Cybern. 45 (2) (2015) 229-241.

[20] H. Li, Y. Pan, Q. Zhou, Filter design for interval type-2 fuzzy systems with D stability constraints under a unified frame, IEEE Trans. Fuzzy Syst. 23 (3) (2015) 719-725.

[21] K. Tanaka, T. Hori, H.O. Wang, A fuzzy Lyapunov approach to fuzzy control system design. in: Proc. American Control Conf., 2001, pp. 4790-4795.

[22] K. Tanaka, T. Hori, H.O. Wang, A multiple Lyapunov function approach to stabilization of fuzzy control systems, IEEE Trans. Fuzzy Syst. 11 (4) (2003) 582-589.

[23] J.C. Doyle, K. Glover, P.P. Khargonekar, B.A. Francis, State-space solutions to standard $H_{2}$ and $H_{\infty}$ control problems, IEEE Trans. Autom. Control 34 (8) (1989) 831-847.

[24] C. Lin, Q. Wang, T.H. Lee, $H_{\infty}$ output tracking control for nonlinear systems via T-S fuzzy model approach, IEEE Trans. Syst., Man, Cybern. 36 (2) (2006) 450-457.

[25] J. Qiu, G. Feng, H. Gao, Static-output-feedback $H_{\infty}$ control of continuous-time T-S fuzzy affine systems via piecewise Lyapunov functions, IEEE Trans. Fuzzy Syst. 21 (2) (2013) 245-261. 
[26] J. Qiu, H. Tian, Q. Lu, H. Gao, Nonsynchronized robust filtering design for continuoustime T-S fuzzy affine dynamic systems based on piecewise Lyapunov functions, IEEE Trans. Cybern. 43 (6) (2013) 1755-1766.

[27] Y. Mao, H. Zhang, Exponential stability and robust $H_{\infty}$ control of a class of discretetime switched non-linear systems with time-varying delays via T-S fuzzy model, Int. J. Syst. Sci. 45 (5) (2014) 1112-1127.

[28] H. Liu, X. Lin, Finite-time $H_{\infty}$ control for a class of nonlinear system with time-varying delay, Neurocomputing 149 (3) (2015) 1481-1489.

[29] J. Qiu, S.X. Ding, H. Gao, S. Yin, Fuzzy-model-based reliable static output feedback $H_{\infty}$ control of nonlinear hyperbolic PDE systems, IEEE Trans. Fuzzy Syst. DOI 10.1109/TFUZZ.2015.2457934.

[30] T. Wang, S. Tong, $H_{\infty}$ control design for discrete-time switched fuzzy systems, Neurocomputing 151 (5) (2015) 782-789.

[31] T. Wang, H. Gao, J. Qiu, A combined adaptive neural network and nonlinear model predictive control for multirate networked industrial process control, IEEE Trans. Neur. Net. Lear. syst. doi: 10.1109/TNNLS.2015.2411671.

[32] J. Qiu, Y. Wei, H.R. Karimi, New approach to delay-dependent $H_{\infty}$ control for continuous-time Markovian jump systems with time-varying delay and deficient transition descriptions, Journal of the Franklin Institute 352 (1) (2015) 189-215. 


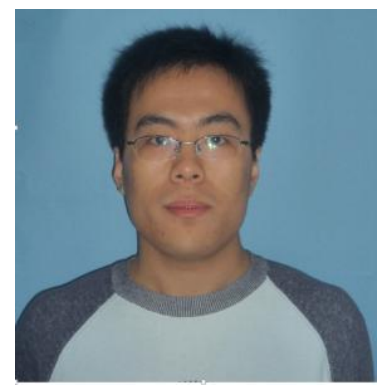

Qunxian Zheng received the B.E. degree in biomedical engineering and M.S. degree in biophysics from University of Electronic Science and Technology of China, Chengdu, in 2008 and 2011, respectively. He is currently working towards his PhD degree in circuits and systems at the University of Electronic Science and Technology of China, Chengdu. His research interests include fuzzy control and switched systems.

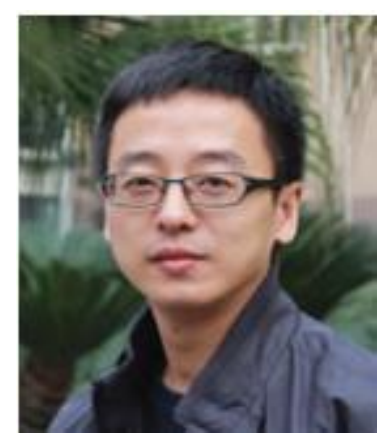

Hongbin Zhang received the BEng degree in aerocraft design from Northwestern Polytechnical University, Xi' an, China, in 1999, and the MEng and PhD degrees in circuits and systems from the University of Electronic Science and Technology of China, Chengdu, in 2002 and 2006, respectively. He has been with the School of Electrical Engineering, University of Electronic Science and Technology of China, since 2002, where he is currently a professor. From August 2008 to August 2010, he has served as a research fellow with the Department of Manufacturing Engineering and Engineering Management, City University of Hong Kong, Kowloon, Hong Kong. His current research interests include intelligent control, autonomous cooperative control and integrated navigation. 